\title{
THE CLASSIFICATION OF COMPACT SURFACES BY USING BERGER'S LEMMA
}

\author{
Masao MAEDA
}

Dedicated to Professor Tominosuke Otsuki on his 75 th birthday

(Received October 29, 1991)

(Revised January 29, 1992)

\section{Introduction.}

Let $M$ be a compact Riemannian manifold and $p, q \in M$ are the points such that

$$
d(p, q)=d(M) \quad(:=\text { diameter of } M)
$$

where $d$ is the distance function of $M$. Then in [1], M. Berger gave the following interesting fact which is known as Berger's Lemma:

FACT (M. Berger). For any non-zero tangent vector $v \in T_{p}(M)$, there exists a shortest geodesic $\gamma:[0, d(p, q)] \rightarrow M$ from $p$ to $q$ satisfying

$$
\Varangle(v, \dot{\gamma}(0)) \leqq \frac{\pi}{2} .
$$

Here $T_{p}(M)$ denotes the tangent space to $M$ at $p$ and $\Varangle(x, y)$ the angle between the vectors $x$ and $y$. In this note, all geodesics are parametrized by arc-length. By $G(p, q)$, we will denote the set of all shortest geodesics from $p$ to $q$.

Now the purpose of this note is to show that all compact surfaces which is homeomorphic to a 2-dimensional sphere $S^{2}$ can be classified into three types. To this classification, we will use the conditions considerd by using Berger's Lemma.

\section{Three conditions.}

For a compact surface $M$, we will consider the following three conditions 
$[\mathrm{C} 0],[\mathrm{CI}],[\mathrm{CII}]:$

[C0] For any pair of points $p, q \in M$ satisfying $d(p, q)=d(M)$ and any non-zero vector $v \in T_{p}(M)$, there exists a geodesic $\gamma \in G(p, q)$ such that

$$
\Varangle(v, \dot{\gamma}(0)) \leqq \frac{\pi}{2}
$$

[CI ] For any pair of points $p, q \in M$ satisfying $d(p, q)=d(M)$, any subset $G^{\prime}(p, q) \subset G(p, q)$ which satisfies the following condition $(*)$ :

(*) For any non-zero vector $v \in T_{p}(M)$, there exists some $\gamma \in G^{\prime}(p, q)$ such that $\Varangle(v, \dot{\gamma}(0)) \leqq \frac{\pi}{2}$

also satisfies the following condition $(* *)$ :

(**) For any non-zero tangent vector $w \in T_{q}(M)$, there exists some $\gamma \in G^{\prime}(p, q)$ such that $\Varangle\left(w,-\dot{\gamma}(d(p, q)) \leqq \frac{\pi}{2}\right.$.

[CII] For any pair of points $p, q \in M$ satisfying $d(p, q)=d(M)$, following condition $(* * *)$ is satisfied:

$(* * *)$ For any two non-zero tangent vectors $v \in T_{p}(M)$ and $w \in T_{q}(M)$, there exists a geodesic $\gamma \in G(p, q)$ such that $\Varangle(v, \dot{\gamma}(0)) \leqq \frac{\pi}{2}$ and $\Varangle(w,-\dot{\gamma}(d(p, q))) \leqq \frac{\pi}{2}$.

Of course by Berger's Lemma, condition [C0] is satisfied automorphically for all compact surfaces. Condition [CI] and [CII] are considered in connection with Berger's Lemma and are related mutually. Lemma.

For later use, we will give a Proposition which is equivalent to Berger's

Proposition 1. Let $p, q \in M$ are the points satisfying $d(p, q)=d(M)$. Then there exists a subset $G^{\prime}(p, q) \subset G(p, q)$ whose number of elements equais 2 or 3 and satisfying the following condition:

for any non-zero vector $v \in T_{p}(M)$, there exists some $\gamma \in G^{\prime}(p, q)$ such that $\Varangle(v, \dot{\gamma}(0)) \leqq \frac{\pi}{2}$.

This can be seen easily but we will give a proof here because the method used in the proof of this Proposition is useful later. 
Proof of Proposition 1. Let $\gamma_{1}:[0, d(p, q)] \rightarrow M$ be a shortest geodesic from $p$ to $q$. Then by Berger's Lemma, putting $v=-\dot{\gamma}_{1}(0)$, we get another geodesic $\gamma_{2} \in G(p, q)$ such that

$$
\Varangle\left(v, \dot{\gamma}_{2}(0)\right) \leqq \frac{\pi}{2} .
$$

If $\Varangle\left(-\dot{\gamma}_{1}(0), \dot{\gamma}_{2}(0)\right)=0$ i.e. $-\dot{\gamma}_{1}(0)=\dot{\gamma}_{2}(0)$, then $G^{\prime}(p, q)=\left\{\gamma_{1}, \gamma_{2}\right\}$ satisfies the required condition. In the case $\Varangle\left(-\dot{\gamma}_{1}(0), \dot{\gamma}_{2}(0)\right)>0$, putting $v=-\left(\dot{\gamma}_{1}(0)\right.$ $\left.+\dot{\gamma}_{2}(0)\right)$ we get a geodesic $\gamma_{3} \in G(p, q)$ such that

$$
\Varangle\left(v, \dot{\gamma}_{3}(0)\right) \leqq \frac{\pi}{2} .
$$

If $\Varangle\left(\dot{\gamma}_{1}(0), \dot{\gamma}_{2}(0)\right)+\Varangle\left(\dot{\gamma}_{2}(0), \dot{\gamma}_{3}(0)\right) \geqq \pi, G^{\prime}(p, q)=\left\{\gamma_{1}, \gamma_{2}, \gamma_{3}\right\}$ is the one which we want. If $\Varangle\left(\dot{\gamma}_{1}(0), \dot{\gamma}_{2}(0)\right)+\Varangle\left(\dot{\gamma}_{2}(0), \dot{\gamma}_{3}(0)\right)<\pi$, then putting $v=-\left(\dot{\gamma}_{1}(0)+\dot{\gamma}_{3}(0)\right)$ we also get a geodesic $\gamma_{4} \in G(p, q)$ such that

$$
\Varangle\left(v, \dot{\gamma}_{4}(0)\right) \leqq \frac{\pi}{2} .
$$

If $\Varangle\left(\dot{\gamma}_{1}(0), \dot{\gamma}_{2}(0)\right)+\Varangle\left(\dot{\gamma}_{2}(0), \dot{\gamma}_{4}(0)\right) \geqq \pi$, then $G^{\prime}(p, q)=\left\{\gamma_{1}, \gamma_{2}, \gamma_{4}\right\}$ is the one which we want. If $\Varangle\left(\dot{\gamma}_{1}(0), \dot{\gamma}_{2}(0)\right)+\Varangle\left(\dot{\gamma}_{2}(0), \dot{\gamma}_{4}(0)\right)<\pi$, then putting $v=-\left(\dot{\gamma}_{1}(0)+\dot{\gamma}_{4}(0)\right)$, we also get a geodesic $\gamma_{5} \in G(p, q)$ such that

$$
\Varangle\left(v, \dot{\gamma}_{5}(0)\right) \leqq \frac{\pi}{2} .
$$

Repeating this process we can reduce to the following two cases:

Case 1. There exists a sequence of geodesics $\left\{\gamma_{i}\right\}_{i=1,2, \ldots, m} \subset G(p, q)$

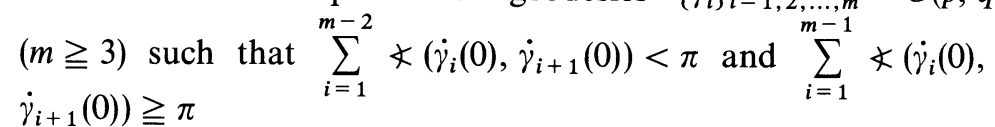

Case 2. There exists a sequence of geodesics $\left\{\gamma_{i}\right\}_{i=1,2,3, \ldots} \subset G(p, q)$ such that $\sum_{i=1}^{k} \Varangle\left(\dot{\gamma}_{i}(0), \dot{\gamma}_{i+1}(0)\right)<\pi$ for all $k$ and $\sum_{i=1}^{\infty} \Varangle\left(\dot{\gamma}_{i}(0), \dot{\gamma}_{i+1}(0)\right)$

If case 1 occur, then letting $G^{\prime}(p, q)=\left\{\gamma_{1}, \gamma_{2}, \gamma_{m}\right\}$ and if case 2 occur, letting $G^{\prime}(p, q)=\left\{\gamma_{1}, \gamma_{2}, \gamma_{\infty}\right\}$, we complete the proof of Proposition 1 where $\gamma_{\infty} \in G(p, q)$ is the limitting geodesic of $\left\{\gamma_{i}\right\}$.

Q.E.D. 
Proposition 1 is an improved version of Berger's Lemma.

In the following section, we will give an example of surface which does not satisfy condition $[\mathrm{CI}]$. Thus condition $[\mathrm{CI}]$ is strictly stronger than condition $[\mathrm{CO}]$.

To investigate the difference between condition [CI] and [CII], we now further assume that $M$ is homeomorphic to a 2-dimensional sphere $S^{2}$.

Let $p, q \in M$ are points satisfying $d(p, q)=d(M)$. Then by two geodesics $\gamma_{1}, \gamma_{2} \in G(p, q), M$ is devided into two disjoint domains $D$ and $D^{\prime}$ such that

$$
\partial D=\partial D^{\prime}=\gamma_{1}([0, d(p, q)]) \cup \gamma_{2}([0, d(p, q)]) .
$$

In this situation, we will call $D$ as an $H$-domain if it satify the following conditions :

$$
\Varangle_{D}\left(\dot{\gamma}_{1}(0), \dot{\gamma}_{2}(0)\right) \leqq \pi \quad \text { and } \quad \Varangle_{D}\left(-\dot{\gamma}_{1}(d(p, q)),-\dot{\gamma}_{2}(d(p, q))>\pi\right.
$$

or

$$
\Varangle_{D}\left(\dot{\gamma}_{1}(0), \dot{\gamma}_{2}(0)\right)>\pi \quad \text { and } \quad \Varangle_{D}\left(-\dot{\gamma}_{1}(d(p, q)),-\dot{\gamma}_{2}(d(p, q))\right) \leqq \pi .
$$

Here $\Varangle_{D}($,$) denotes the inner angle measured on D$.

LEMMA 1. A compact surface $M$ which is homeomorphic to $S^{2}$ satisfies condition [CI] if and only if there exists no $H$-domain in $M$.

Proof. Assume $M$ satisfy condition [CI]. If there exist $H$-domain $D$ such that for certain $\gamma_{1}, \gamma_{2} \in G(p, q)$

$$
\partial D=\gamma_{1}([0, d(p, q)]) \cup \gamma_{2}([0, d(p, q)])
$$

and

$$
\Varangle_{D}\left(\dot{\gamma}_{1}(0), \dot{\gamma}_{2}(0)\right)>\pi, \Varangle_{D}\left(-\dot{\gamma}_{1}(d(p, q)),-\dot{\gamma}_{2}(d(p, q))\right) \leqq \pi .
$$

Then by the argument in the proof of Proposition 1, we can get another geodesic $\gamma_{3} \in G(p, q)$ such that $\gamma_{3}((0, d(p, q))) \subset D$ and

$$
\Varangle_{D}\left(\dot{\gamma}_{1}(0), \dot{\gamma}_{3}(0)\right) \leqq \pi, \Varangle_{D}\left(\dot{\gamma}_{2}(0), \dot{\gamma}_{3}(0)\right) \leqq \pi .
$$

Thus the set $G^{\prime}(p, q)=\left\{\gamma_{1}, \gamma_{2}, \gamma_{3}\right\} \subset G(p, q)$ satisfies the assumption (*) in [CI]. Hence by $[\mathrm{CI}], G^{\prime}(p, q)=\left\{\gamma_{1}, \gamma_{2}, \gamma_{3}\right\}$ must satisfy the condition $(* *)$. But this is impossible, because $\gamma_{3}((0, d(p, q))) \subset D$ and hence we have

$$
\Varangle\left(-\dot{\gamma}_{1}(d(p, q)),-\dot{\gamma}_{3}(d(p, q))\right) \leqq \Varangle_{D}\left(-\dot{\gamma}_{1}(d(p, q)),-\dot{\gamma}_{3}(d(p, q))\right) \leqq \pi
$$


and

$$
\Varangle\left(-\dot{\gamma}_{2}(d(p, q)),-\dot{\gamma}_{3}(d(p, q))\right) \leqq \Varangle_{D}\left(-\dot{\gamma}_{2}(d(p, q)),-\dot{\gamma}_{3}(d(p, q))\right) \leqq \pi .
$$

To show the converse, let $G^{\prime}(p, q) \subset G(p, q)$ be any subset which satisfies the condition $(*)$ in $[\mathrm{CI}]$. Assuming that this $G^{\prime}(p, q)$ does not satisfy the condition $(* *)$, we will derive a contradiction. Since $G^{\prime}(p, q)$ does not satisfy $(* *)$, we can find a half circle $\widetilde{S}^{1}$ in $T_{q}^{1}(M):=\left\{v \in T_{p}(M):\|v\|(:=\right.$ norm of $\left.v)=1\right\}$ such that

$$
\left\{-\dot{\gamma}(d(p, q)): \gamma \in G^{\prime}(p, q)\right\} \subset \tilde{S}^{1}
$$

and the number of elements of the set

$$
\partial \tilde{S}^{1} \cap\left\{-\dot{\gamma}(d(p, q)): \gamma \in G^{\prime}(p, q)\right\}
$$

is at most one. Here we are identifying $T_{q}^{1}(M)$ with a circle $S^{1}$.

Now take a geodesic $\gamma_{1} \in G^{\prime}(p, q)$ and put $v:=-\dot{\gamma}_{1}(0)$. Then from the condition $(*)$, we can find another geodesic $\gamma_{2} \in G^{\prime}(p, q)$ with $\Varangle\left(-\dot{\gamma}_{1}(0), \dot{\gamma}_{2}(0)\right)$ $\leqq \pi / 2$. If $\dot{\gamma}_{2}(0)=-\dot{\gamma}_{1}(0)$, then the subset $G^{\prime \prime}(p, q)=\left\{\gamma_{1}, \gamma_{2}\right\} \subset G^{\prime}(p, q)$ $\subset G(p, q)$ satisfies $(*)$. If $\Varangle\left(\dot{\gamma}_{1}(d(p, q)), \dot{\gamma}_{2}(d(p, q))\right)<\pi$, then we can easily find an $H$-domain. So it must be

$$
\Varangle\left(\dot{\gamma}_{1}(d(p, q)), \dot{\gamma}_{2}(d(p, q))\right)=\pi .
$$

Thus $G^{\prime \prime}(p, q)$ satisfies the condition $(* *)$ and hence $G^{\prime}(p, q)$ satisfies $(* *)$. This is a contradiction.

In the case $\Varangle\left(\dot{\gamma}_{1}(0), \dot{\gamma}_{2}(0)\right)<\pi$, by the argument in the proof of Proposition 1, we can find third geodesic $\gamma_{3} \in G^{\prime}(p, q)$ such that $G^{\prime \prime}(p, q)$ $:=\left\{\gamma_{1}, \gamma_{2}, \gamma_{3}\right\}$ satisfies the condition $(*)$. Then since

$$
\left\{\dot{\gamma}_{i}(d(p, q)): i=1,2,3\right\} \subset\left\{\dot{\gamma}(d(p, q)): \gamma \in G^{\prime}(p, q)\right\} \subset \tilde{S}^{1}
$$

we can easily get an $H$-domain and this is a contradiction.

q.e.d.

Using this Lemma 1, we can show the following

Proposition 2. Let $M$ be a compact surface which is homeomorphic to $S^{2}$ and satisfies condition [CII]. Then $M$ satisfies condition [CI] also.

In the following section, we will give an example of compact surfaces which satisfies condition [CI] but does not satisfy condition [CII]. Thus condition $[\mathrm{CII}]$ is strictly stronger than $[\mathrm{CI}]$. 
Proof of Proposition 2. Assume $M$ does not satisfy condition [CI]. Then by Lemma 1 , there exists a pair of points $p, q \in M$ satisfying $d(p, q)=d(M)$ and $H$-domain $D$ such that

$$
\partial D=\gamma_{1}([0, d(p, q)]) \cup \gamma_{2}([0, d(p, q)])
$$

and

$$
\begin{aligned}
& \Varangle_{D}\left(\dot{\gamma}_{1}(0), \dot{\gamma}_{2}(0)\right) \leqq \pi, \\
& \Varangle_{D}\left(-\dot{\gamma}_{1}(d(p, q)),-\dot{\gamma}_{2}(d(p, q))\right)>\pi
\end{aligned}
$$

where $\gamma_{1}, \gamma_{2} \in G(p, q)$. Then putting $v:=-\left(\dot{\gamma}_{1}(0)+\dot{\gamma}_{2}(0)\right), w:=\dot{\gamma}_{1}(d(p, q))$ $+\dot{\gamma}_{2}(d(p, q))$, by $[\mathrm{CII}]$ we get a geodesic $\gamma_{3} \in G(p, q)$ satisfying

$$
\Varangle\left(v, \dot{\gamma}_{3}(0)\right) \leqq \pi / 2 \text { and } \Varangle\left(w, \dot{\gamma}_{3}(d(p, q)) \leqq \pi / 2 .\right.
$$

Then since $\dot{\gamma}_{3}(d(p, q)) \neq \dot{\gamma}_{1}(d(p, q))$ and $\dot{\gamma}_{3}(d(p, q)) \neq \dot{\gamma}_{2}(d(p, q))$, we know that $\gamma_{3} \neq \gamma_{1}, \gamma_{2}$ and $\gamma_{3}([d(p, q)-\varepsilon, d(p, q))) \subset D$ for some $\varepsilon>0$. Also we know that $\gamma_{3}\left(\left(0, \varepsilon^{\prime}\right]\right) \subset \bar{D}^{C}$ for some $\varepsilon^{\prime}>0$. Thus $\gamma_{3} \mid\left[\varepsilon^{\prime}, d(p, q)-\varepsilon\right]$ is connecting a point $\left(=\gamma_{3}\left(\varepsilon^{\prime}\right)\right)$ in $\bar{D}^{C}$ to a point $\left(=\gamma_{3}(d(p, q)-\varepsilon)\right)$ in $D$. Thus using the assumption that $M$ is homeomorphic to $S^{2}$, we see $\gamma_{3} \mid\left[\varepsilon^{\prime}, d(p, q)-\varepsilon\right]$ must intersect $\partial D=\gamma_{1}([0, d(p, q)]) \cup \gamma_{2}([0, d(p, q)])$ which is an image of simply closed curve $\gamma_{2}^{-1} \circ \gamma_{1}$. This contradicts the shortestness of $\gamma_{1}$ or $\gamma_{2}$.

Q.E.D.

Now we will call a compact surface $M$ homeomorphic to $S^{2}$ to be of type 0 if $M$ does not satisfy condition [CI]. Note that condition [C0] is satisfied for all compact Riemannian manifold automorphically by Berger's Lemma. Similarly we will call $M$ to be of type 1 (resp. type 2) if $M$ satisfies condition $[\mathrm{CI}]$ but does not satisfy condition [CII] (resp. satisfies condition [CII]). So putting

$$
m:=\left\{M: M \text { is a compact surface homeomorphic to } S^{2}\right\}
$$

and

$$
m_{i}:=\{M: M \in m \text { and is of type } i\} \quad i=0,1,2,
$$

we can summarize Proposition 1 and Proposition 2 as

THEOREM 1. $m=m_{0} \cup m_{1} \cup m_{2}$ 
REMARK. Each $m_{i}$ is non-empty as is shown in the following section.

\section{Examples.}

In this section, we will give an example of compact surfaces of each type $i, i=0,1,2$.

$1^{\circ}$. Type 2 examples.

A standard sphere $S^{2}$ in a Euclidean 3 -space $E^{3}$ is an example of surface which is of type 2. More generally, let $M$ be a compact surface of revolution in $E^{3}$ which is homeomorphic to $S^{2}$. If its two vertices $p, q \in M$ satisfy $d(p, q)=d(M)$ and

$$
d(p, q)>d\left(p^{\prime}, q^{\prime}\right) \quad \text { for all }\left(p^{\prime}, q^{\prime}\right)(\neq(p, q)) \in M \times M,
$$

then $M$ is also of type 2 . As such examples, we can take ellipsoids

$$
x^{2}+y^{2}+\frac{z^{2}}{k^{2}}=1 \quad(k>1)
$$

where $(x, y, z)$ denotes standard orthogonal coordinates of $E^{3}$.

$2^{\circ}$. Type 1 examples.

As exampes of this type, we also take ellipsoids

$$
\frac{x^{2}}{a^{2}}+\frac{y^{2}}{b^{2}}+\frac{z^{2}}{c^{2}}=1 \quad(0<a<b<c) .
$$

The diameter of these surfaces are attained for the pair of points $(p, q)$ such that $p=(0,0, c), q=(0,0,-c)$ and $G(p, q)$ consists of exactly two shortest geodesics $\gamma_{1}, \gamma_{2}$ with $\dot{\gamma}_{1}(0)=-\dot{\gamma}_{2}(0), \dot{\gamma}_{1}(d(p, q))=-\dot{\gamma}_{2}(d(p, q))$. So these surfaces satisfy the condition [CI] but not satisfy condition [CII] and hence of type 1.

$3^{\circ}$. Type 0 examples.

In $E^{3}$, we consider two rectangles $R_{1}$ and $R_{2}$ whose four vertices are

$$
\begin{aligned}
& R_{1}:(0,0,0),(0, a, 0),(0, a, b),(0,0, b) \\
& R_{2}:(1,0,0),(1, a, 0),(1, a, b),(1,0, b)
\end{aligned}
$$

and four half cylinders $C_{1}, C_{2}, C_{3}, C_{4}$ which are defined by the following 
equations :

$$
\begin{aligned}
& C_{1}:(x-1 / 2)^{2}+(z-b)^{2}=1, z \geqq b, y+z \geqq b,-y+z \geqq b-a \\
& C_{2}:(x-1 / 2)^{2}+z^{2}=1, z \leqq 0,-y+z \leqq 0, y+z \leqq a \\
& C_{3}:(x-1 / 2)^{2}+y^{2}=1, y \leqq 0, y+z \leqq b,-y+z \geqq 0 \\
& C_{4}:(x-1 / 2)^{2}+(y-a)^{2}=1, y \geqq a,-y+z \leqq b-a, y+z \geqq a
\end{aligned}
$$

where $a, b$ are sufficiently large constants satifying $0<a<b$. Let $S$ be a compact surface obtained by attaching four half cylinder $C_{1} \sim C_{4}$ with $R_{1}$ and $R_{2}$. Obviously this flat surface is homeomorphic to $S^{2}$ and has singularities. Furthermore $S$ can be considered as a metric space whose metric $d$ is induced from the flat metric. Then $S$ has a center $s_{\circ}(1 / 2, a / 2, b / 2)$ i.e. there exists an isometry $\sigma: S \rightarrow S$ induced from the central symmetry $\Sigma: E^{3} \rightarrow E^{3}$ with respect to $s_{\circ}$. Then we can check that

$$
d(S)(=\text { diameter of } S)=d\left(p_{\circ}, q_{\circ}\right)
$$

where $p_{\circ}=(1 / 2,-1, b+1), q_{\circ}=(1 / 2, a+1,-1)$. Note that the points $p_{\circ}, q_{\circ} \in S$ satisfy $q_{\circ}=\sigma\left(p_{\circ}\right)\left(p_{\circ}=\sigma\left(q_{\circ}\right)\right)$. Furthermore we can see that there exist four shortest curves $\gamma_{1}, \gamma_{2}, \gamma_{3}, \gamma_{4}$ connecting $p_{\circ}$ to $q_{0}$. Let $\gamma_{1}$ and $\gamma_{2}$ (resp. $\gamma_{3}$ and $\gamma_{4}$ ) are those which are contained in the half space $\{x \geqq 1 / 2\}$ (resp. $\{x \leqq 1 / 2\})$ in $E^{3}$. We can assume that $\sigma\left(\gamma_{1}\right)=\gamma_{3}$ and $\sigma\left(\gamma_{2}\right)=\gamma_{4}$. Since $S$ is symmetric under the reflection $t$ with respect to the plane $\{y=a / 2\}$, we also have points $p_{1}=t\left(p_{\circ}\right), q_{1}=t\left(q_{\circ}\right)$ satisfying $d\left(p_{1}, q_{1}\right)=d(S)$ and shortest curves $\sigma_{i}\left(=t\left(\gamma_{i}\right)\right), i=1,2,3,4$ connecting from $p_{1}$ to $q_{1}$.

Now, we will regularize $S$ so as to obtain a smooth convex surface $\widetilde{S} \subset E^{3}$ satisfying the following conditions:

(i) For a fixed small $\varepsilon>0, S-\{\varepsilon-$ tubular neighborhood of four half circles $S_{i}, i=1,2,3,4$ in $\left.S\right\}$ is imbedded isometrically in $\tilde{S}$ as a flat surface. Here $S_{i}$ is defined by the following equations:

$$
\begin{aligned}
& S_{1}:(x-1 / 2)^{2}+(z-b)^{2}=1, y+z=b, z \geqq b \\
& S_{2}:(x-1 / 2)^{2}+(z-b)^{2}=1,-y+z=b-a, z \geqq b \\
& S_{3}:(x-1 / 2)^{2}+z^{2}=1,-y+z=0, z \leqq 0 \\
& S_{4}:(x-1 / 2)^{2}+z^{2}=1, y+z=a, z \leqq 0
\end{aligned}
$$

(in the following, we will add further restrictions). 
(ii) In each $\varepsilon$-neighborhood of $S_{i}, S$ is regularized symmetrically with respect to $S_{i}$.

(iii) $\tilde{S}$ has a centre $s_{\circ}=(1 / 2, a / 2, b / 2)$ i.e. there exists an isometry $\sigma: \tilde{S}-\tilde{S}$ induced from the central symmetry $\Sigma: E^{3}-E^{3}$ with respect to $s_{\circ}$.

(iv) There exists a point $\tilde{p}_{\circ}$ (resp. $\left.\tilde{q}_{\circ}\right) \in \widetilde{S}$ near $p_{\circ}\left(\right.$ resp. $\left.q_{\circ}\right) \in S$ such that $\tilde{p}_{\circ}, \tilde{q}_{\circ}$ are contained in the plane $\{x=1 / 2\} \subset E^{3}$ and $d\left(\tilde{p}_{\circ}, \tilde{q}_{\circ}\right)=d(\widetilde{S})$.

(v) There exists four shortest geodesics $\tilde{\gamma}_{i}$ near $\gamma_{i}, i=1,2,3,4$ connecting from $\tilde{p}_{\circ}$ to $\tilde{q}_{\circ}$ such that $\tilde{\gamma}_{1}$ and $\tilde{\gamma}_{2}$ (resp. $\tilde{\gamma}_{3}$ and $\tilde{\gamma}_{4}$ ) are contained in the half space $\{x \geqq 1 / 2\}$ (resp. $\{x \leqq 1 / 2\})$ and

$$
\sigma\left(\tilde{\gamma}_{1}\right)=\tilde{\gamma}_{3}, \quad \sigma\left(\tilde{\gamma}_{2}\right)=\tilde{\gamma}_{4} .
$$

Let $K$ denotes the Gaussian curvature of $S$. Then, by construction, $K$ takes non-zero values only in a small neighborhood $V\left(\tilde{p}_{\circ}\right)\left(\operatorname{resp} . V\left(\tilde{q}_{\mathrm{c}}\right), V\left(\tilde{p}_{1}\right), V\left(\tilde{q}_{1}\right)\right.$ ) of point $\tilde{p}_{\circ}$ (resp. $\left.\tilde{q}_{\circ}, \tilde{p}_{1}, \tilde{q}_{1}\right) . \quad \tilde{p}_{1}, \tilde{q}_{1} \in \tilde{S}$ are the points near $p_{1}$ and $q_{1}$ respectively satisfying $d\left(\tilde{p}_{1}, \tilde{q}_{1}\right)=d(\tilde{S})$.

Now for a subset $U \subset \tilde{S}$, let denote the integral curvature of $U$ by $c(U)$ :

$$
c(U)=\int_{U} K d \tilde{S}
$$

where $d \tilde{S}$ is the area element of $\tilde{S}$. Since $c(U)$ equals the area of image $g(U)$ of $U$ of the Gauss map $g: \tilde{S} \rightarrow S^{2} \subset E^{3}$, we see

$$
c\left(V\left(\tilde{p}_{\circ}\right)\right)=c\left(V\left(\tilde{q}_{\circ}\right)\right)=c\left(V\left(\tilde{p}_{1}\right)\right)=c\left(V\left(\tilde{q}_{1}\right)\right) .
$$

Thus from Gauss-Bonnet's Theorem, we have

$$
c\left(V\left(\tilde{p}_{i}\right)\right)=c\left(V\left(\tilde{q}_{i}\right)\right)=\pi, \quad i=1.2 .
$$

Put $\alpha:=\Varangle\left(\dot{\tilde{\gamma}}_{1}(0), \dot{\tilde{\gamma}}_{2}(0)\right)$ and $\beta:=\Varangle\left(\dot{\gamma}_{1}\left(d\left(p_{\circ}, q_{\circ}\right)\right), \dot{\tilde{\gamma}}_{2}\left(d\left(p_{\circ}, q_{\circ}\right)\right)\right)$.

Here as was announced in (i), we will add a further restriction on $\tilde{S}$. Namely, we regularize $S$ along $S_{1}$ and $S_{4}$ so as to have

$$
\alpha \neq \beta \text {. }
$$

Let $D_{1}$ be the domain bounded by $\tilde{\gamma}_{1}$ and $\tilde{\gamma}_{2}$ satisfying $\alpha=\Varangle_{D_{1}}\left(\dot{\gamma}_{1}(0), \dot{\tilde{\gamma}}_{2}(0)\right)$.

Then since $\beta \leqq \pi$,

$$
\beta=\Varangle_{D_{1}}\left(\dot{\tilde{\gamma}}_{1}\left(d\left(\tilde{p}_{\circ}, \tilde{q}_{\circ}\right)\right), \dot{\tilde{\gamma}}_{2}\left(d\left(\tilde{p}_{\circ}, \tilde{q}_{\circ}\right)\right)\right) .
$$

By symmetry, we also have 


$$
\beta=\Varangle\left(\dot{\tilde{\gamma}}_{3}(0), \dot{\tilde{\gamma}}_{4}(0)\right) \text {. }
$$

So if we put

$$
\begin{aligned}
& \delta:=\Varangle\left(\dot{\tilde{\gamma}}_{2}(0), \dot{\tilde{\gamma}}_{3}(0)\right), \\
& \mu:=\Varangle\left(\dot{\tilde{\gamma}}_{2}\left(d\left(\tilde{p}_{\circ}, \tilde{q}_{\circ}\right)\right), \dot{\gamma}_{3}\left(d\left(\tilde{p}_{\circ}, \tilde{q}_{\circ}\right)\right)\right),
\end{aligned}
$$

then $\delta=\mu$ and

$$
\begin{aligned}
& \mu=\Varangle\left(\dot{\tilde{\gamma}}_{1}(0), \dot{\tilde{\gamma}}_{4}(0)\right), \\
& \delta=\Varangle\left(\dot{\tilde{\gamma}}_{1}\left(d\left(\tilde{p}_{\circ}, \tilde{q}_{\circ}\right)\right), \tilde{\tilde{\gamma}}_{4}\left(d\left(\tilde{p}_{\circ}, \tilde{q}_{\circ}\right)\right)\right) .
\end{aligned}
$$

Thus we can find geodesics $\tilde{\gamma}_{i^{\circ}}$ and $\tilde{\gamma}_{j_{\circ}}, i_{\circ} \neq j_{\circ}$ bounding an $H$-domain.

\section{Number of shortest geodesics.}

In the previous sections, we showed that all compact surfaces which are homeomorphic to a 2-dimensional sphere $S^{2}$ are classified into three types and there exist examples of each type certainly. In its classification, the set $G(p, q)$ plays important roles. So in this section, we will study certain properties of $G(p, q)$.

Let $M$ be a compact surface homeomorphic to a 2-dimensional sphere $S^{2}$ and $p, q \in M$ the points satisfying $d(p, q)=d(M)$.

(0). Type 0 case.

In proposition 1 , we saw that

$$
\# G(p, q) \geqq 2
$$

where \# denotes the number of elements. If \# $G=2$, then $G(p, q)=\left\{\gamma_{1}, \gamma_{2}\right\}$ and its composite $\gamma_{2}^{-1} \circ \gamma_{1}$ is a simple closed geodesic through $p$ and $q$. Thus $G(p, q)$ satisfies condition [CI]. If \# $G(p, q)=3$, let $G(p, q)=\left\{\gamma_{1}, \gamma_{2}, \gamma_{3}\right\}$. Then $G(q, p)=\left\{\gamma_{1}^{-1}, \gamma_{2}^{-1}, \gamma_{3}^{-1}\right\}$. Thus $G(p, q)$ satisfies condition [CI]. So if $M$ is of type 0 , then for some pair of points $p, q \in M$ satisfying $d(p, q)=d(M)$, it must hold that

$$
\text { \# } G(p, q) \geqq 4 \text {. }
$$

In section 2 , we gave an example of surfaces of type 0 with $G(p, q)$ satisfying

$$
\# G(p, q)=4 \text {. }
$$


REMARK. It may be an interesting problem whether there exist an example of compact surface of type 0 with two pair of points $p, q \in M$ and $\tilde{p}, \tilde{q} \in M$ such that $d(p, q)=d(M), d(\tilde{p}, \tilde{q})=d(M)$ and satisfying

$$
\text { \# } G(p, q) \geqq 4, \quad \# G(\tilde{p}, \tilde{q}) \leqq 3 .
$$

(2). Type 1 case.

In section 2, we gave an example of surfaces such that \#G(p,q)=2 for all pair of points $p, q \in M$ with $d(p, q)=d(M)$ (In fact, such pair of points $p, q \in M$ was unique). Thus for type 1 surface, we can not obtain a further sharp estimate other than

$$
\# G(p, q) \geqq 2
$$

(3). Type 2 case.

As is stated in section 2, a standard 2-dimensional sphere $S^{2}$ is an example of surface of type 2. In this example, each geodesic $\gamma:[0, d(p, q)] \rightarrow S^{2}$ starting from $p$ belongs to $G(p, q)$ and hence

$$
\# G(p, q)=\infty
$$

where $p, q \in S^{2}$ are the points satisfying $d(p, q)=d\left(S^{2}\right)$. We will show that this phenomenon is occuring for all compact surfaces of type 2 .

Let $M$ be a compact surface of type 2 and $p, q \in M$ are the points satisfying $d(p, q)=d(M)$. We identify $T_{p}^{1}\left(=\left\{v \in T_{p}(M):\|v\|=1\right)\left(\operatorname{resp} . T_{q}^{1}(M)\right)\right.$ with the standard unit circle $S^{1}=[0,2 \pi](2 \pi$ is identified with 0$)$. This identification is done so as to satisfy the following conditions:

(i) We fix a geodesic $\gamma_{1} \in G(p, q)$ and identify $\dot{\gamma}_{1}(0)$ with $0 \in S^{1}$ (resp. $-\dot{\gamma}_{1}(d(p, q))$ with $\left.0 \in S^{1}\right)$.

(ii) For other geodeisc $\gamma_{2} \in G(p, q)$, if $\Varangle_{+}\left(\dot{\gamma}_{1}(0), \dot{\gamma}_{2}(0)\right)$, the angle between $\dot{\gamma}_{1}(0)$ and $\dot{\gamma}_{2}(0)$ measured with respect to the positive orientation on $T_{p}(M)$, equals to $\alpha$, then

$$
\dot{\gamma}_{2}(0)=\alpha \in S^{1}
$$

(resp. if $\Varangle_{D}\left(-\dot{\gamma}_{1}(d(p, q)),-\dot{\gamma}_{2}(d(p, q))\right)=\beta$, then $-\dot{\gamma}_{2}(d(p, q))=\beta \in S^{1}$ where $D$ is the domain in $M$ bounded by $\gamma_{2}^{-1} \circ \gamma_{1}$ such that $\left.\Varangle_{D}\left(\dot{\gamma}_{1}(0), \dot{\gamma}_{2}(0)\right)=\alpha\right)$. Here we note that two domaines bounded by $\gamma_{2}^{-1} \circ \gamma_{1}$ are not $H$-domains. To avoid the confusion, we denote the circle $T_{p}^{1}(M)$ (resp. $T_{q}^{1}(M)$ ) by $S^{1}(p)$ (resp. $\left.S^{1}(q)\right)$. Then $T_{p}^{1}(M) \times T_{q}^{1}(M)$ can be considerd as a flat torus $S^{1}(p) \times S^{1}(q)$. For a point $(s, t) \in S^{1}(p) \times S^{1}(q)$, we use the notation $(s, t) \in G(p, q)$. This 
means that there exists a geodesic $\gamma \in G(p, q)$ satisfying $\dot{\gamma}(0)=s$ and $-\dot{\gamma}(d(p, q))=t$.

We need some lemmas.

Lemma 2 (Uniqueness). For any $s \in S^{1}(p)$, there exists at most one $t \in S^{1}(q)$ such that

$$
(s, t) \in G(p, q) .
$$

This follows from the uniqueness of geodesic for the initial values.

Lemma 3 (Continuity). If a sequence $\left\{\left(s_{i}, t_{i}\right)\right\}_{i=1,2, \ldots} \subset G(p, q)$ converge to $\left(s_{\circ}, t_{\circ}\right) \in S^{1}(p) \times S^{1}(q)$, then $\left(s_{\circ}, t_{\circ}\right) \in G(p, q)$.

Proof. Let $\gamma_{i} \in G(p, q)$ such that $\dot{\gamma}_{i}(0)=s_{i}$ and $-\dot{\gamma}_{i}(d(p, q))=t_{i}$ for $i=1,2,3, \ldots$ Then the limit geodesic $\gamma:=\lim _{i \rightarrow \infty} \gamma_{i}$ satisfies $\dot{\gamma}(0)=s_{\circ},-\dot{\gamma}(d(p, q))$ $=t_{\circ}$ and $\gamma \in G(p, q)$.

Lemma 4 (Monotonicity). For $(s, t)$ and $\left(s^{\prime}, t^{\prime}\right) \in G(p, q)$, if $s<s^{\prime}$ then $t<t^{\prime}$.

Proof. Let $\gamma$ and $\sigma \in G(p, q)$ are the geodesics such that $\dot{\gamma}(0)=s$, $-\dot{\gamma}(d(p, q))=t$ and $\dot{\sigma}(0)=s^{\prime},-\dot{\sigma}(d(p, q))=t^{\prime}$ respectively. Since $\gamma$ and $\sigma$ are shortest geodesics, $\gamma$ and $\sigma$ do not intersect except at $p$ and $q$. Thus if $D$ (resp. $D^{\prime}$ ) denote the domain bounded by $\gamma^{-1} \circ \gamma_{1}$ (resp. $\sigma^{-1} \circ \gamma_{1}$ ) such that $\Varangle_{D}\left(\gamma(0), \gamma_{1}(0)\right)=s\left(\right.$ resp. $\left.\Varangle_{D^{\prime}}\left(\dot{\sigma}(0), \dot{\gamma}_{1}(0)\right)=s^{\prime}\right)$, then

$$
D \subset D^{\prime} .
$$

Thus we know

$$
\Varangle_{D}\left(-\dot{\gamma}(d(p, q)),-\dot{\gamma}_{1}(d(p, q)) \leqq \Varangle_{D^{\prime}}\left(-\dot{\sigma}(d(p, q)),-\dot{\gamma}_{1}(d(p, q))\right)\right.
$$

i.e. $t<t^{\prime}$.

q.e.d.

Now putting $S_{\circ}^{1}(p):=\left\{s \in S^{1}(p):(s, t) \in G(p, q)\right.$ for some $\left.t \in S^{1}(q)\right\}\left(\subset S^{1}(p)\right)$, we will prove the following:

Theorem 2. For type 2 surface $M, S_{\circ}^{1}(p)=S^{1}(p)$ holds i.e. all geodesics starting from $p$ are shortest connections to $q$ with length $d(M)$ where $p \in M$ and $q \in M$ are the points satisfying $d(p, q)=d(M)$.

Proof. We will consider $S^{1}(p) \times S^{1}(q)$ as is obtained from the Euclidean plane $E^{2}$ by $\bmod 2 \pi$ identification for each variable. All argument in the 
following are done under $\bmod 2 \pi$ identification if we do not state otherwise.

At first we note that for each $\left(s_{\circ}, t_{\circ}\right) \in G(p, q)$ and for any $(s, t) \in\left[s_{\circ}-\pi / 2\right.$, $\left.s_{\circ}+\pi / 2\right] \times\left[t_{\circ}-\pi / 2, t_{\circ}+\pi / 2\right]$, there exists some $(\tilde{s}, \tilde{t}) \in G(p, q)$ such that

$$
\Varangle(s, \tilde{s}) \leqq \pi / 2 \text { and } \Varangle(t, \tilde{t}) \leqq \pi / 2
$$

because we can take such $(\tilde{s}, \tilde{t})$ as $(\tilde{s}, \tilde{t})=\left(s_{\circ}, t_{\circ}\right)$. Here $\Varangle(s, \tilde{s}) \leqq \pi / 2$ (also $\Varangle(t, \tilde{t}) \leqq \pi / 2)$ means for the geodesics $\gamma, \tilde{\gamma} \in G(p, q)$ such that $\dot{\gamma}(0)=s, \dot{\tilde{\gamma}}(0)=\tilde{s}$, it holds $\Varangle(\dot{\gamma}(0), \dot{\gamma}(0)) \leqq \pi / 2$.

Conversely, since $G(p, q)$ satisfies condition [CII], for any $(s, t) \in S^{1}(p) \times S^{1}(q)$, there exists $(\tilde{s}, \tilde{t}) \in G(p, q)$ satisfying

$$
(s, t) \in[\tilde{s}-\pi / 2, \tilde{s}+\pi / 2] \times[\tilde{t}-\pi / 2, \tilde{t}+\pi / 2] .
$$

Thus to show the assertion, it suffices to show that if $S_{\circ}^{1}(p) \subsetneq S^{1}(p)$, then

$$
S^{1}(p) \times S^{1}(q) \varnothing \bigcup_{s \in S_{\circ}^{1}(p)}[s-\pi / 2, s+\pi / 2] \times[t-\pi / 2, t+\pi / 2] .
$$

Here $t \in S^{1}(q)$ is the one such that $(s, t) \in G(p, q)$. So assuming $S_{\circ}^{1}(p) \subsetneq S^{1}(p)$ and $S^{1}(p) \times S^{1}(q) \subset \bigcup_{s \in S_{\circ}^{1}(p)}[s-\pi / 2, s+\pi / 2] \times[t-\pi / 2, t+\pi / 2]$, we will derive a contradiction. Then since $S_{\circ}^{1}(p) \subsetneq S^{1}(p)$, there exist $s_{1}, s_{2} \in S_{\circ}^{1}(p)$ such that $s \notin S_{\circ}^{1}(p)$ for any $s \in\left(s_{1}, s_{2}\right)$. This follows from Lemma 2 and Lemma 3 . Let $t_{1}, t_{2} \in S^{1}(q)$ satisfy $\left(s_{1}, t_{1}\right) \in G(p, q)$ and $\left(s_{2}, t_{2}\right) \in G(p, q)$. To simplify the argument, for $s \in S_{\circ}^{1}(p)$, put $I_{s}:=[s-\pi / 2, s+\pi / 2] \times[t-\pi / 2, t+\pi / 2]$, where $t \in S^{1}(q)$ is the one satisfying $(s, t) \in G(p, q)$. Since the continuous curve $c$ defined on $S_{\circ}^{1}(p)$ by $c(s)=(s, t), s \in S_{\circ}^{1}(p)$ where $t \in S^{1}(q)$ is the one satisfying $(s, t) \in G(p, q)$ does not defined on the interval $\left(s_{1}, s_{2}\right) \subset S^{1}(p)$, so terminal point $c\left(s_{1}\right)$ of $c$ jumps to terminal point $c\left(s_{2}\right)$ of $c$ as $s$ varies from $s_{1}$ to $s_{2}$ because of Lemma 3 and Lemma 4 . So rectangle $I_{s}$ jumps also from $I_{s_{1}}$ to $I_{s_{2}}$ as $s$ varies from $s_{1}$ to $s_{2}$. So as $a$ point characterizing this jump from $I_{s_{1}}$ to $I_{s_{2}}$, we choose the point $(\hat{s}, \hat{t}):=\left(s_{1}+\pi / 2, t_{2}-\pi / 2\right) \in S^{1}(p) \times S^{1}(q)$. Note that $\pi / 2 \leqq \hat{s} \leqq 5 \pi / 2$ and $-\pi / 2 \leqq \hat{t} \leqq 3 \pi / 2$.

Furthermore we have

LEMMA 5. $\bigcup_{s \in S_{\circ}^{1}(p)} I_{s} \subset[-\pi / 2,5 \pi / 2] \times[-\pi / 2,5 \pi / 2]$.

This follows from Lemma 4 and the fact that $(0,0)=(2 \pi, 2 \pi) \in G(p, q)$ i.e. $0=2 \pi \in S_{\circ}^{1}(p)$. 
LEMMA 6. (i). $(\hat{s}, \hat{t}) \notin(\pi / 2,5 \pi / 2) \times(3 \pi / 2,5 \pi / 2)$,

(ii) $(\hat{s}, \hat{t}) \notin(-\pi / 2, \pi / 2) \times(-\pi / 2,5 \pi / 2)$.

(i) and (ii) follows from the fact that $\hat{t} \leqq 3 \pi / 2$ and $\hat{s} \geqq \pi / 2$ respectively.

LEMma 7. $(\hat{s}, \hat{t}) \notin(\pi / 2,3 \pi / 2) \times(\pi / 2,3 \pi / 2)$.

Proof. If $(\hat{s}, \hat{t}) \in(\pi / 2,3 \pi / 2) \times(\pi / 2,3 \pi / 2)$, then for sufficiently small $\varepsilon>0,(\hat{s}+\varepsilon, \hat{t}-\varepsilon) \in(\pi / 2,3 \pi / 2) \times(\pi / 2,3 \pi / 2)$ and $(\hat{s}+\varepsilon, \hat{t}-\hat{\varepsilon}) \notin \bigcup_{s \in S_{0}^{1}(p)} I_{s}$ because $I_{s_{1}}$ jumps to $I_{s_{2}}$ as $s$ varies from $s_{1}$ to $s_{2}$ and any point of $(\pi / 2,3 \pi / 2)$ $\times(\pi / 2,3 \pi / 2)$ can not be coverd twice by the rectangles in $\left\{I_{s}\right\}_{s \in S_{\circ}^{1}(p)}$ from Lemma 5 . q.e.d.

LEMMA 8. $(\hat{s}, \hat{t}) \notin(3 \pi / 2,5 \pi / 2) \times(-\pi / 2, \pi / 2)$.

Proof. Consider another point $\left(\hat{s}^{\prime}, \hat{t}^{\prime}\right):=\left(s_{2}-\pi / 2, t_{1}+\pi / 2\right) \in S^{1}(p) \times S^{1}(q)$ characterizing the jump from $I_{s_{1}}$ to $I_{s_{2}}$. If $(\hat{s}, \hat{t}) \in(3 \pi / 2,5 \pi / 2) \times(-\pi / 2, \pi / 2)$, then we can check easily that $\left(\hat{s}^{\prime}, \hat{t}^{\prime}\right) \in(\pi / 2,3 \pi / 2) \times(\pi / 2,3 \pi / 2)$. Thus for sufficiently small $\varepsilon>0$, it holds $\left(\hat{s}^{\prime}-\varepsilon, \hat{t}^{\prime}+\varepsilon\right) \in(\pi / 2,3 \pi / 2) \times(\pi / 2,3 \pi / 2)$ and $\left(\hat{s}^{\prime}-\varepsilon, \hat{t}^{\prime}+\varepsilon\right) \notin \bigcup_{s \in S_{\circ}^{1}(p)} I_{s}$ because $I_{s_{1}}$ jumps to $I_{s_{2}}$ also. Then by the same reason in the proof of Lemma 7, this leads to a conradiction. q.e.d.

LEMMA 9. $(\hat{s}, \hat{t}) \notin(\pi / 2,3 \pi / 2) \times(-\pi / 2, \pi / 2)$.

Proof. If $(\hat{s}, \hat{t}) \in(\pi / 2,3 \pi / 2) \times(-\pi / 2, \pi / 2)$, then we can check that another point $\left(\hat{s}^{\prime}, \hat{t}^{\prime}\right)$ characterizing the jump from $I_{s_{1}}$ to $I_{s_{2}}$ satisfies $\left(\hat{s}^{\prime}, \hat{t}^{\prime}\right) \in(-\pi / 2,3 \pi / 2) \times(\pi / 2,3 \pi / 2)$. But just as the proof of Lemma 7 , we can show that $\left(\hat{s}^{\prime}, \hat{t}^{\prime}\right) \notin[\pi / 2,3 \pi / 2) \times(\pi / 2,3 \pi / 2)$. Thus it sufficies to show that $\left(\hat{s}^{\prime}, \hat{t}^{\prime}\right) \notin(-\pi / 2, \pi / 2) \times(\pi / 2,3 \pi / 2)$. So assuming that $\left(\hat{s}^{\prime}, \hat{t}^{\prime}\right) \in(-\pi / 2, \pi / 2)$ $\times(\pi / 2,3 \pi / 2)$, we will derive a contradiction. Then since there is a jump from $I_{s_{1}}$ to $I_{s_{2}}$, for sufficiently small $\varepsilon>0$,

$$
\begin{aligned}
& (\hat{s}+\varepsilon, \hat{t}-\varepsilon) \in(\pi / 2,3 \pi / 2) \times(-\pi / 2, \pi / 2) \\
& \left(\hat{s}^{\prime}-\varepsilon, \hat{t}^{\prime}+\varepsilon\right) \in(-\pi / 2, \pi / 2) \times(\pi / 2,3 \pi / 2)
\end{aligned}
$$

and

$$
\begin{aligned}
& (\hat{s}+\varepsilon, \hat{t}-\varepsilon) \notin \bigcup_{s \leqq s_{2}, s \in S_{\circ}^{1}(p)} I_{s} \\
& \left(\hat{s}^{\prime}-\varepsilon, \hat{t}^{\prime}+\varepsilon\right) \notin \bigcup_{s \leqq s_{2}, s \in S_{\circ}^{1}} I_{s} .
\end{aligned}
$$


Furthermore we add another condition on $\varepsilon$ such that $\hat{s}^{\prime}-\hat{s}+\pi>2 \varepsilon$. This is possible because $0<\hat{s}-\hat{s}^{\prime}<\pi$. Thus since $S^{1}(p) \times S^{1}(q) \subset \bigcup_{s \in S_{0}^{1}(p)} I_{s}$, there exists $\bar{s}, \bar{s}^{\prime} \in S_{\circ}^{1}(p)$ satisfying $(\hat{s}+\varepsilon, \hat{t}-\varepsilon+2 \pi) \in I_{\bar{s}}$ and $\left(\hat{s}^{\prime}-\varepsilon+2 \pi, \hat{t}^{\prime}+\varepsilon\right) \in I_{\bar{s}}$, because $\pi / 2<\hat{s}+\varepsilon<3 \pi / 2$ and $\pi / 2<\hat{t}^{\prime}+\varepsilon<3 \pi / 2$. Let $\bar{t}, \bar{t}^{\prime} \in S^{1}(q)$ are the elements satisfying $(\bar{s}, \bar{t}) \in G(p, q)$ and $\left(\bar{s}^{\prime}, \bar{t}^{\prime}\right) \in G(p, q)$. Thus

$$
|(\hat{s}+\varepsilon)-\bar{s}| \leqq \pi / 2,|(\hat{t}-\varepsilon)+2 \pi-\bar{t}| \leqq \pi / 2
$$

and

$$
\left|\left(\hat{s}^{\prime}-\varepsilon\right)+2 \pi-\bar{s}^{\prime}\right| \leqq \pi / 2,\left|\left(\hat{t}^{\prime}+\varepsilon\right)-\bar{t}\right| \leqq \pi / 2 .
$$

From this, we get $s<s^{\prime}$ and $t^{\prime}>t$. But this contradicts to Lemma 4 . q.e.d.

Similarly we can check the following:

LEMMA $10 . \quad(\hat{s}, \hat{t}) \notin(3 \pi / 2,5 \pi / 2) \times(\pi / 2,3 \pi / 2)$.

Finally by the proof of Lemma 9 , we can see that $\hat{s} \neq \pi / 2,3 \pi / 2$ and $\hat{t} \neq \pi / 2,3 \pi / 2$.

Thus summurizing the above Lemmas, we know

$$
(\hat{s}, \hat{t}) \notin S^{1}(p) \times S^{1}(q) .
$$

This is a contradiction and hence we proved $S_{\circ}^{1}(p)=S^{1}(p)$.

Q.E.D.

\title{
References
}

[1] M. Berger; Sur quelques varietés riemanniennes suffisament pincees, Bull. Soc. Math. France, 88, 57-71 (1960).

[2] B. C. Croke; Area and the length of the shortest closed geodesic, Jour. Diff. Geom. 27, 1-21 (1988).

[3] D. Gromoll, W. Klingengerg and W. Meyer; Riemannsche Geometrie im Grossen, Springer-Verlag, 1968.

[4] T. SAKAI; On the isodiametric inequlity for the 2-sphere, Geometry of Manifolds, Perspectives in Math. Academic Press, 303-315

The auther would like to express his hearty thanks to the referee of this paper for his many valuable suggestions.

\author{
Department of Mathematics \\ Faculty of Education \\ Yokohama National University
}

